\title{
NIA workshop on senescence in brain aging and Alzheimer's disease and its related dementias
}

\author{
Amanda M. DiBattista • Felipe Sierra • \\ Eliezer Masliah
}

Received: 3 December 2019 / Accepted: 3 January 2020/Published online: 13 January 2020

(C) This is a U.S. government work and not under copyright protection in the U.S.; foreign copyright protection may apply 2020

\section{Introduction}

Understanding Alzheimer's disease and its related dementias (AD/ADRD) in the context of aging is crucial toward developing new treatments and elucidating pathogenic AD/ADRD mechanisms. However, there is a gap in our understanding of how senescence may distinguish healthy brain aging from neurodegenerative disease, including AD/ADRD. Most published studies on cellular senescence were conducted in tissues outside of the brain, making it unclear whether these processes are conserved in the brain. While acute cell cycle arrest in senescent cells promotes normal development and physiological functions such as wound healing outside of the brain, chronically, senescent cells are both metabolically active and resistant to apoptosis. As chronically senescent cells accumulate over time, they release a variety of pro-inflammatory factors - referred to as the senescence-associated secretory phenotype (SASP) that influence surrounding cells. The SASP is

A. M. DiBattista $(\bowtie) \cdot$ E. Masliah

Division of Neuroscience, National Institute on Aging, Bethesda, MD, USA

e-mail: amanda.dibattista@nih.gov

E. Masliah

e-mail: eliezer.masliah@nih.gov

F. Sierra

Division of Aging Biology, National Institute on Aging, Bethesda, MD, USA

e-mail: sierraf@mail.nih.gov hypothesized to influence neighboring non-senescent cells by changing their physiology, including facilitating their conversion to senescent cells. Because senescence has been well-studied in the context of peripheral tissues and longevity, the National Institute on Aging (NIA) organized a workshop to address senescence in brain aging and $\mathrm{AD} / \mathrm{ADRD}$. This workshop addressed the goals of the NIA strategic directions for research on aging by seeking to improve our understanding of the aging brain, Alzheimer's disease, and other neurodegenerative diseases (National Institute on Aging 2016). It also relates to $\mathrm{AD} / \mathrm{ADRD}$ Research Implementation Milestones to integrate the study of fundamental biology of aging with neurobiology of aging and research on neurodegeneration, $\mathrm{AD}$ and $\mathrm{AD}$-related dementias to better understand the mechanisms of vulnerability and resilience in $\mathrm{AD}$ (https://www.nia.nih.gov/research/ milestones).

\section{Workshop organization}

The NIA publicly announced a planned workshop to address the potential effects of cell senescence in brain aging and AD/ADRD in the May 2019 Director's Status Report presented at the National Advisory Council on Aging. On September 18-19, 2019, approximately 30 participants from NIA and the broader research community came together for the live webcast day-and-a-half of meetings held in Bethesda, MD. The main goals of the workshop were to assess the state of knowledge and science in the field of cellular senescence, highlight 
challenges researchers face that may be preventing the field from moving forward, and identify areas in which further support is needed to facilitate progress. The workshop was organized into three sessions addressing the following key: (1) Systemic Factors, Senescence, and Brain Aging; (2) Non-Neuronal Cells, Senescence, and Brain Aging; and (3) Senescence in Alzheimer's Disease and Its Related Dementias.

Brad Wise, Ron Kohanski, and Amanda DiBattista from the NIA welcomed participants and opened the workshop with brief remarks about its motivation and logistics. Brad Wise, Ph.D., Acting Deputy Director and Chief of the Neurobiology of Aging Branch of NIA's Division of Neuroscience, described the pathological pathways underlying brain aging and the progression of Alzheimer's disease and its related dementias (AD/ ADRD) as an area that warrants further investigation. Senescence has been well-studied in the context of peripheral tissues and longevity, Dr. Wise noted, but less so in brain aging and $\mathrm{AD} / \mathrm{ADRD}$. Interest in this subject has grown, he added, as a result of studies suggesting that senescent cells may be an effective target for new therapeutic approaches. Recent scientific papers have reported, for example, that senolytic therapy can reduce cognitive decline in animal models of AD/ADRD. Ron Kohanski, Ph.D., Deputy Director of the Division of Aging Biology at NIA, explained the geroscience perspective of how various hallmarks of aging (e.g., chromosome maintenance and DNA repair, senescence, inflammation, proteostasis, immune and metabolic dysregulation, and others) interact in ways that are beneficial or deleterious to human health under different circumstances. Genotype and cell type are important parts of the equation, and the Division of Aging Biology interacts closely with the Division of Neuroscience, Dr. Kohanski explained, because neurons are a unique subset of cell types, functioning in a special environment of their own. Amanda DiBattista, Ph.D., Program Director in the Division of Neuroscience, provided an overview of the challenges in understanding cellular senescence in the context of the brain. Throughout the duration of the workshop, Dr. DiBattista encouraged participants to provide insight on several questions facing the field of cell senescence during healthy and pathological brain aging.

- What is it about the aging process that induces senescence? How does it occur in the brain compared with the rest of the body? Could senescence outside of the brain drive brain senescence?
- Could senescent cells be beneficial for the brain in some ways? Similarly, are there any processes for which eliminating senescent cells in the brain could be detrimental?

- Is there a link between lifestyle (i.e., diet and exercise) and senescence, and could this affect senescence in the brain?

- What are the properties of senescent cells in the brain? Could biomarkers of senescent cells also represent biomarkers of aging (and vice versa)?

- How do senescent cells interact with non-senescent cells in the brain? Could decreasing one senescent cell type decrease the number of other senescent cell types by interfering with the general spread of senescence?

- Can post-mitotic cells (e.g., neurons) undergo a senescence-like phenotype in the human brain? If so, what are the effects on non-neuronal cells? Moreover, what variations in senescence are there within and across brain cell types?

- Can senescence in AD be studied without consideration for aging? Can $\mathrm{AD}$ and aging be studied in parallel, or do they need to be studied together?

- Does senescence contribute to $\mathrm{AD}$, or does $\mathrm{AD}$ contribute to senescence? Can we answer this question using currently available tools/resources?

- Is there a role for targeting a dynamic process like senescence in precision medicine? Is it possible to target only part of the SASP, for example? If so, which part should be targeted?

\section{Session summaries}

Session I: systemic factors, senescence, and brain aging

Jan van Deursen, Ph.D. of the Mayo Clinic, provided a brief overview of cellular senescence for context, then described a number of in vivo models (in mice) that are helping to advance research in the field. Though cellular senescence plays a critical role in biological mechanisms such as tissue repair and cancer immunosurveillance, he explained, the process evolved through natural selection to serve people in their reproductive period; with the "natural lifespan" now greatly extended, the process breaks down in later years. Senescent cells accumulate over time in tissues and organs, and despite being in a state of cell cycle arrest, ancillary 
SASP factors, released in response to various stressors, can interact with neighboring cells and contribute to common age-related diseases. Similar SASP-induced effects are applicable to the brain, Dr. van Deursen noted, with senescent cells releasing factors affecting the activity of astrocytes, microglia, and oligodendrocytes in ways that aid the progression of neurodegenerative diseases. Research to date has suggested that senescent cells could be involved in multiple diseases of aging, including dementia, atherosclerosis, and osteoarthritis. By clearing senescent cells or preventing their accumulation, senolytics offer a potentially useful strategy to combat diseases of aging, much like tackling cancer. The field of senolytics must proceed cautiously, Dr. van Deursen stressed, because knowledge of senescent cells in vivo is limited and much remains to be learned about underlying mechanisms and possible side effects (van Deursen 2019). A major challenge to researchers looking for answers, he noted, is that senescent cells are not easy to detect and control.

Viviana Perez, Ph.D. of Oregon State University, described research findings that help explain how cellular senescence can play a role in the development of Alzheimer's disease. Recent studies in her lab have focused heavily on understanding the mechanisms of the drug rapamycin, which has been shown to inhibit cell senescence in vivo and extend longevity in mice, including mouse models of AD. The researchers are now investigating the transcription factor Nrf2, which regulates expression of antioxidant proteins that protect against oxidative damage triggered by injury and inflammation. Dr. Perez said that the findings point to a central role for $\mathrm{Nrf} 2$ in processes such as oxidative stress, inflammatory response, and proteostasis that "prime" the brain for cellular senescence in Alzheimer's. Future directions, she proposed, should include a search for analogs of rapamycin with better permeability to the blood-brain barrier. There is also a need, she added, for studies that consider cell type specificity, and that lead to greater knowledge of cellular mechanisms involved in this avenue of research. For example, some neuronal cell types are always positive for senescence-associated $\beta$-galactosidase (SA $\beta$-gal) staining, while the marker is reversible in others.

Thomas Foster, Ph.D. of the University of Florida, described the work from his lab exploring the question of whether systemic inflammation contributes to agerelated cognitive impairment. Low-level increases in cellular and molecular markers of brain aging begin to emerge in middle age, which led the researchers to search for markers for systemic inflammation in the blood, hippocampus, and cortex of young, middle-aged, and aged mice. Dr. Foster said that the findings from these and related studies suggest that chronic systemic inflammation likely contributes to age-related cognitive impairment and brain senescence. As to whether systemic inflammation may induce brain aging, much like senescence, the answer depends on the acute or chronic nature of that inflammation. Systemic inflammation may be "priming" the system, Dr. Foster explained, making the brain more susceptible to low-level chronic systemic inflammation of aging. One challenge, he noted, is developing a true chronic model of inflammation that moves beyond multiple acute injections to test its relationship with senescence in the brain.

Irina Conboy, Ph.D. of the University of California, Berkeley, summarized years of research into stem cells, and why they lose their regenerative ability over time. The work involved stitching young and old mice together to exchange blood, in a technique known as heterochronic parabiosis. Tissue senescence is not entirely intrinsic, Dr. Conboy said, and various studies have shown that selective ablation of cells with high levels of p16 can improve health and repair of some- but not all-tissues. P16, a normal CDK inhibitor, is physiologically upregulated during repair of young and old skeletal muscle (Mehdipour et al. 2019). Dr. Conboy said that this heterochronic parabiosis approach of "resetting" pathways synergistically to reverse multiple symptoms of aging has implications for attenuating or preventing a number of degenerative and metabolic diseases as a class instead of approaching each of them individually. She briefly described a variety of tools and bioanalytic techniques (e.g., BONCAT, FUNCAT, Click-A+ Chip) to help identify "young" proteins that are capable of rejuvenating and define age-elevated inhibitors of tissue health and repair. Senescence is multifactorial, she stressed, and blood exchange benefits work by more than one factor; a combination is needed for tissue repair.

Rodney Johnson, Ph.D. of the University of Illinois Urbana-Champaign, presented findings from his lab showing that dietary factors - in this case, dietary fiber-can affect microglial activity central to agerelated neuroinflammation. While microglial-mediated neuroinflammation benefits the brain in many ways - as in immune surveillance, enhanced plasticity, and tissue repair - chronic neuroinflammation has been implicated in brain pathology leading to conditions such as 
cognitive impairment, anxiety and depression, and neuronal damage. If dietary factors can affect age-related neuroinflammation, it leaves the question open of whether dietary and neuro-immune interactions also influence the processes of cellular senescence in the brain.

Session II: non-neuronal cells, senescence, and brain aging

Julie Andersen, Ph.D. of the Buck Institute for Research on Aging, described research exploring how senescence in astrocytes and neurons may contribute to neurodegeneration. The overarching hypothesis is that early amyloid beta $(A \beta)$ pathology induces senescence in neurons and/or astrocytes creating stress-induced senescence in neighboring cells via the SASP. This could allow the spread of pathology independent of $A \beta$, which may explain why its elimination in later stages of disease could be ineffective. Late-stage losses in immune privilege would enable clearance of senescent cells via infiltration of immune cells from the periphery, and large-scale elimination of immune cells at late disease stage may not be entirely beneficial and, in fact, could be detrimental (Walton \& Andersen 2019). By relying on early markers of senescence, she explained, researchers may not be detecting the full cohort of senescent cells at later stages in the process, which could explain the disconnection between the small percentage of senescent cells observed and noted effects of their elimination on disease progression.

Anna Csiszar, M.D., Ph.D. of the University of Oklahoma Health Science Center, described experiments that offer insight into the role that neurovascular senescence that plays in cognitive decline. In the neurovascular unit, astrocytes, neurons, microglia, and cerebromicrovascular endothelial cells (CMVECs) are susceptible to irradiation-induced DNA damage and senescence. To explore these links, Dr. Csiszar and her colleagues developed a model of whole brain irradiation in transgenic p16-3MR mice. Among the key findings, Dr. Csiszar said, the results show that senolytic treatments could be used potentially to reverse irradiationinduced accelerated neurovascular aging, mitigating the adverse effects of cancer treatments. One obstacle for understanding senescence in the brain, she noted, is that several cell type-specific markers-such as GFAPchange over the course of aging.
Michael Clarke, M.D. of Stanford University, presented research from his lab investigating molecular regulation of stem cell self-renewal in relation to $\mathrm{AD} /$ ADRD. Current work has focused heavily on the BMI pathway, flowing from the group's discovery 16 years ago that the protein Bmi-1 plays a key role in selfrenewal. Dr. Clarke was drawn to the $\mathrm{AD} / \mathrm{ADRD}$ field, he said, after $\mathrm{AD}$ mice were found to have, even at a young age, a defect that results in hyperproliferation of neural stem cells. Part of a polycomb ring finger complex, Bmi-1 is a master regulator of several major proteins (Cdkn2a, p16, p19, etc.), and promotes ubiquitination of histones and chromatin remodeling. Though studying stem cell self-renewal rather than neurodegeneration, the researchers discovered that Usp16, a modulator of ubiquitination, inhibits self-renewal of neural progenitor cells. It is unknown whether eliminating quiescent cells using senolytic approaches could affect already declining rates of neurogenesis in the aging brain by enabling progenitor cells to self-renew.

Kotb Abdelmohsen, Ph.D. of NIA's Intramural Program, described a variety of techniques his lab is using to detect new markers of senescence - surface-ome, proteome, transcriptome, and secretome - and to test senotherapy approaches that might eliminate or control those targets to prevent their detrimental effects, thereby improving brain health. In one set of studies involving mouse and human brain tissue, the researchers found high populations of senescent oligodendrocyte precursor cells (OPCs) in human $\mathrm{A} \beta$ plaques, and improved AD phenotype when treating with senolytics to eliminate senescent OPCs in AD mice (Zhang et al. 2019). The researchers are currently testing how surface proteins expressed on senescent cells - known as the senescent "surface-ome" - can be exploited as novel targets for basic science and potential therapeutic approaches.

Session III: senescence in Alzheimer's disease and its related dementias

Darren Baker, Ph.D. of the Mayo Clinic, described the results of research showing that the protein tau, widely implicated in $\mathrm{AD} / \mathrm{ADRD}$, mediates neurodegeneration and senescence. Dr. Baker noted that certain normal age-related processes can be amplified in diseases of aging. To test whether this could be the case for cellular senescence in $\mathrm{AD} / \mathrm{ADRD}$, the researchers began developing a novel animal model. They crossed a tau-based mouse model (MAPT ${ }^{\mathrm{P} 301 \mathrm{~S}}$ PS19) with an ATTAC 
mouse line that allows selective killing of targeted senescent markers upon treatment with AP20187, a synthetic drug. Senescent cells accumulated, accompanied by hyperphosphorylated tau, and promoted reactive gliosis. The drug treatment strategy rescued many of these effects (Bussian et al. 2018). Dr. Baker said that the findings suggest that when high amounts of hyperphosphorylated tau are expressed, elevated stress occurs, and senescent glial cells start to accumulate. This leads, in turn, to promotion of SASP activity as well as more activated glial cells. Cumulatively, all of these factors lead to more tau hyperphosphorylation and subsequent neurodegeneration. The next research steps, he noted, will entail looking at whether attenuation of senescence leads to reduced neurodegeneration.

Wolfgang "Jake" Streit, Ph.D. of the University of Florida, described findings from human brain tissue studies that help explain the role of microglia in $\mathrm{AD} /$ ADRD and senescence. Dr. Streit argues amyloid formation continues while microglia attempt phagocytosis in $\mathrm{AD}$, but by the time end-stage plaques appear, microglia become exhausted and dystrophic. This microglial dystrophy develops concurrently with tau pathology, Dr. Streit said, and $A \beta$ deposition generally occurs later in life, when tau pathology is already well developed. While they may be part of the normal aging process, these dystrophic microglia are unable to carry out their normal cellular functions and have properties of senescent cells. Future directions include acquiring a better understanding of the causes and mechanisms of microglial dystrophy and its relationship to cellular senescence in the brain.

Erin Hascup, Ph.D. of Southern Illinois University School of Medicine, described research exploring the interaction of cellular senescence, inflammation, and neurotransmission as it relates to brain aging and $\mathrm{AD} /$ ADRD. The work in her lab, she said, stems in part from a cellular senescence hypothesis positing that soluble and insoluble $A \beta$ activates the innate immune system, leading to a self-reinforcing cycle of pro-inflammatory signaling and cellular senescence, which in turn leads to neurodegeneration and cognitive decline. The lab has further studies underway to investigate the mechanisms that might be driving interaction between the three aspects in relation to factors such as aging, glial dysfunction, reactive astrogliosis, pro-inflammatory cytokines, and decreased glutamate transport.

Marcia Gordon, Ph.D. of Michigan State University, summarized a variety of mouse and human transcriptomic studies from her lab to describe the relationship between cellular senescence in aging and $\mathrm{AD} /$ ADRD. While the findings may indicate new markers of senescence to follow in the future, Dr. Gordon said that they also illustrate the complexities in trying to compare results from animal models and human AD pathology. In summary, Dr. Gordon cautioned against bulk analysis of senescent cells that may be small in number and therefore masked in total RNA approaches. Dr. Gordon also warned that neurodegeneration can affect cellular composition, compromising the ability to detect gene changes. Future directions, she added, should include studies comparing brain regions with and without $\mathrm{AD}$ pathology, and the impact of senescence on the function of different cell types in the brain.

Miranda Orr, Ph.D. of Wake Forest School of Medicine, described findings from her studies showing that aggregation of tau protein induces cellular senescence in the brain. Dr. Orr's experiments have found that dasatinib and quercetin mediate clearance of NFT-containing neurons, and significantly lower SASP factors without affecting canonical inflammatory cell types (Musi et al. 2018). Even so, Dr. Orr explains that it is difficult to identify the precise targets of senolytic and senomorphic drugs. Dr. Orr hypothesizes that NFTs induce a senescent-like state, which contributes to neurodegeneration. Her ongoing projects include exploring the mechanisms underlying these effects and tracking the influence of neuronal senescence throughout disease.

\section{Challenges, gaps, and opportunities}

Defining senescence in the brain

At present, there is no single definition of "cellular senescence" that the field can apply across tissues and cell types. Without clear molecular markers or properties that define senescence in the brain, it is difficult to move forward to develop specific targets related to senescence for drug discovery and development for age-related diseases, including Alzheimer's disease and its related dementias (AD/ADRD). This is a particularly relevant gap in understanding as several workshop participants suggested that many of the markers associated with senescence that change with aging worsen in the AD brain. Moreover, while the discussion focused on the downsides of senescence, it was acknowledged that senescence could be beneficial to the 
brain in certain contexts, complicating the rationale for therapeutically targeting senescent cells. Some workshop participants suggested that it may be an example of antagonistic pleiotropy, in which processes underlying senescence in the brain are beneficial early in life, but detrimental later in life. Others pointed out that senescent cells could have an important role in immunosurveillance and repair in the brain. To resolve these and other outstanding questions in the field, there is an opportunity to validate markers of senescence, promote methodological transparency and reproducibility, and develop a clear functional definition of senescence in the brain rather than one that relies on cellular markers as a proxy.

While workshop participants expressed that the optimal definition for senescence would be a functional definition, current research approaches require the use of markers as a proxy for senescence in the brain. However, identifying reliable markers for senescent cells in basic neurobiology research has been a challenge for the field. This is in part because it is difficult to validate markers of senescence in vivo across cell types when each cell type could have a unique senescent signature. For instance, while SA $\beta$-gal frequents lists of senescent cell markers, its levels change with duration of cellular stress and cell type. Some neuronal subtypes are positive for SA $\beta$-Gal staining at nearly every age and stress level, suggesting that it is not an exclusive senescence marker. It can also be challenging to distinguish between true hallmarks of senescence and those of neuroinflammation or normal aging processes. Taking these issues into consideration, participants emphasized the importance of methodological transparency to promote reproducibility in the field. Research groups relying on protein versus transcriptional reporters, or using different antibodies, for example, could have disparate results that have more to do with approach than the validity of a marker for senescence. Armed with this knowledge, however, researchers have the opportunity to promote reproducibility if they understand methodological differences across labs.

Although reliance on markers as a proxy for senescence in the brain may be necessary in practice, workshop participants also emphasized the value of developing a functional definition of senescence. Cellular senescence is a phenomenon that pushes a cell to become dysfunctional in a way that cannot be narrowly defined, in part, because these hallmarks of senescence are disputed. Another challenge in functionally defining senescence in vivo comes from its in vitro origin story. In 1961, Hayflick and Moorhead acknowledged that the phenomenon of limited cell divisions in vitro was a concept "although vague at the level of the whole organism, may have some validity in explaining the phenomenon at the cellular level" (Hayflick \& Moorhead 1961). It can be problematic to try to directly apply the in vitro Hayflick phenomenon in vivo, especially when comparing different cell and tissue types while accounting for their interactions with one another. Though the irreversible nature of senescent cells was challenged by some workshop participants, others maintained that true senescence requires a permanent state of cell-cycle arrest reminiscent of what is observed in vitro. Unable to undergo apoptosis, the prolonged effects of senescent cells are unique in that they persist in aging in a way that is distinct from non-senescent cells. To reconcile these viewpoints, the workshop participants raised the possibility that senescent cells are a heterogenous population. They suggested that while acute senescence could be involved in short-term processes like wound healing, chronic senescence could be defined by the accumulation of cellular stresses fundamentally altering the functional properties of a senescent cell. Chronically senescent cells, then, could be recognized as harmful and subsequently removed by the immune system in a process that becomes less efficient over the course of aging. This could also represent the process by which the senescent secretome influences neighboring cells in a detrimental way. Workshop participants further suggested that these chronically senescent cells are analogous to neoplastic cells, in that both are not present at birth and do not serve a useful purpose to the tissue. Defining the processes of acute and chronic senescence in the protected environment of the brain, and how they could contribute to age-related neurodegenerative disease, remains an open question.

Developing new tools and resources to study senescence

The challenge of defining senescence in the brain has the potential to be addressed through access to new tools and resources tailored to study this unique population of cells. A broad audience of researchers have become interested in studying senescent cells in a relatively short period of time. While this interest has raised new questions about senescence from diverse fields at a rapid rate, appropriate tools are not yet in place to answer them. In general, basic science tools to properly study 
senescent cells in vitro and in vivo are lacking, especially considering the heterogeneity of senescence across cell types. While it is beginning to emerge that the senescent transcriptome may differ based on tissue type, for example, the extent of these differences is not yet clear, particularly between the brain and the rest of the body. Further complicating data interpretation is that most studies suggest that a small subset of senescent cells-less than $1 \%$ in many cases - impact the overall phenotype in vivo. As a result of the noticeable phenotype, the field continues to search for larger populations of senescent cells in vivo that may be regularly overlooked. This has included novel approaches to detect larger populations of senescent cells through selective enrichment methods. However, it is not yet clear whether the difficulty in identifying larger numbers of senescent cells comes from tool and in vivo marker limitations that prevent detection of the total population of senescent cells present, or whether a very small group of cells is simply impacting the system in a substantial way. A potential solution for this would be to develop better tools to facilitate basic studies on senescence in the brain, and various examples of ways that this could be accomplished were discussed during the workshop.

While workshop participants acknowledged the current limitations of studying senescent cells-including the small number of cells needed to impact phenotype and the heterogeneity of senescent markers based on cell type - they also suggested possible solutions to these challenges. These suggestions focused on in vivo approaches because senescent cells are rejuvenated in culture, making it difficult to effectively study the arrested cell-cycle of senescent cells in vitro for long periods of time. Instead, participants suggested that there is an opportunity to develop new tools to identify and target senescent cells in vivo. A hurdle has been limited access to well-characterized and validated animal models with cell type-specific expression that would enable researchers to understand the beneficial properties of senolytics on subgroups of cells. This includes animal models without off-target effects, particularly in those with target genes that are expressed differently over the course of aging. In addition to animal models, there is also an opportunity to develop effective antibodies targeting hypothesized senescent cell markers that could be applied to various model organisms. This could include established markers of senescent cells, but also new and improved markers identifying senescent cells in vivo across cell types. To overcome these challenges, participants emphasized the importance of continuing to encourage collaboration and communication across scientific disciplines, including across the fields of senescence and neurobiology.

Distinguishing senescence pathways in the brain

As senescence is becoming more widely recognized as an interesting phenomenon related to aging, many of the underlying mechanisms driving senescence in the brain are unclear. Several workshop participants cautioned that without understanding these underlying mechanisms, the application of senolytics as therapeutics for neurodegenerative disorders in humans may be premature. For example, beneficial outcomes could be interpreted as due to senolysis while detrimental outcomes could be dismissed as off-target effects. However, at present, it is not exactly clear how senolytics are having their effects on the brain or how they may be affecting non-senescent cells.

Differentiating between normal aging, pathological aging, and senescence processes was identified by workshop participants as a particular challenge facing the field. Because blood-brain-barrier and neuroinflammatory changes precede pathological changes, initial effects of senescence in the brain could begin in the periphery. While some participants pointed out links between diet and neuroinflammation, it was acknowledged that it is not yet clear whether diet has the propensity to induce senescence in the brain through the same mediators or whether a specific subset of systemic proteins can be transferred to the brain to facilitate this process. Among other factors, senescence is one type of change impacting the secretome during aging. While the secretome of a non-senescent cell also changes over time with aging, there is something specific about a senescent cell that is detrimental to the tissue as a whole. In addition, more broadly, very little is known about how brain aging itself impacts the secretome. Specifically, it is unclear what key factors influence this larger process, and what exactly it is about removing senescent cells that may be beneficial. This is a particular hurdle, because it is unknown how a relatively low number of senescent cells in circulation could affect the secretome, especially considering that not all senescent cell populations have detrimental effects. Adding to this complexity is the evidence described by several workshop participants indicating that senescence tracks more with 
background genotype and/or cell type of origin than the intended inducer of senescence.

To move the field forward, workshop participants suggested the possibility of systematically eliminating specific targets in the pathways hypothesized to be critical to senescence to determine whether a therapeutic effect persists in their absence. Other options proposed included developing studies that are more longitudinal to get a better sense of how senescence and brain aging change together over time. It was noted that traditional approaches generate datasets with too few senescent cells at only one timepoint, creating an opportunity for the field to learn from unbiased longitudinal studies. The importance of developing defined stages of brain aging during which to measure senescence-analogous to defined stages of disease in $\mathrm{AD}$ — was also emphasized by workshop participants.

\section{Conclusions}

Discussions among workshop participants resulted in the identification of several critical needs for enabling progress in the field of senescence in brain aging and $\mathrm{AD} / \mathrm{ADRD}$. These included defining senescence in the brain, developing new tools and resources to study senescence in the brain, and distinguishing senescence pathways in the brain in the context of normal aging and AD/ADRD. Specifically, workshop participants emphasized the importance of (1) developing a clear functional definition of senescence in the brain, (2) validating reliable markers of senescence in vivo across cell types, (3) promoting methodological transparency and reproducibility, (4) validating cell type-specific mouse models of senescence, (5) establishing tools to identify and target senescent cells in the brain, (6) encouraging collaboration across the fields of senescence and neurobiology, (7) promoting longitudinal studies to get a better sense of how senescence and brain aging changes over time, (8) developing defined stages of brain aging during which to measure senescence, (9) differentiating between properties of senescent cells in normal brain aging versus disease, and (10) highlighting the unique qualities and mechanisms of senescent cells that make them detrimental over time.

\section{Compliance with ethical standards}

Disclaimer The participation of these individuals or the materials should not be interpreted as representing the official viewpoint of the US Department of Health and Human Services, the National Institutes of Health, or the National Institute on Aging, except where noted.

\section{References}

Bussian TJ, Aziz A, Meyer CF, Swenson BL, van Deursen JM, Baker DJ (2018) Clearance of senescent glial cells prevents tau-dependent pathology and cognitive decline. Nature 562(7728):578-582

Hayflick L, Moorhead PS (1961) The serial cultivation of human diploid cell strains. Exp Cell Res 25:585-621

Mehdipour M, Etienne J, Chen CC, Gathwala R, Rehman M, Kato C, Liu C, Liu Y, Zuo Y, Conboy MJ, Conboy IM (2019) Rejuvenation of brain, liver and muscle by simultaneous pharmacological modulation of two signaling determinants, that change in opposite directions with age. Aging 11(15): 5628-5645

Musi N, Valentine JM, Sickora KR, Baeuerle E, Thompson CS, Shen Q, Orr ME (2018) Tau protein aggregation is associated with cellular senescence in the brain. Aging Cell 17(6): e12840

National Institute on Aging (2016) Aging Well in the 21st Century: Strategic Directions for Research on Aging. https://www.nia.nih.gov/sites/default/files/2017-07/niastrategic-directions-2016.pdf.

van Deursen JM (2019) Senolytic therapies for healthy longevity. Science 364(6441):636-637

Walton CC, Andersen JK (2019) Unknown fates of (brain) oxidation or UFO: close encounters with neuronal senescence. Free Radic Biol Med 134:695-701

Zhang P, Kishimoto Y, Grammatikakis I, Gottimukkala K, Cutler RG, Zhang S, Abdelmohsen K, Bohr VA, Misra Sen J, Gorospe M, Mattson MP (2019) Senolytic therapy alleviates A $\beta$-associated oligodendrocyte progenitor cell senescence and cognitive deficits in an Alzheimer's disease model. Nat Neurosci 22(5):719-728

Publisher's note Springer Nature remains neutral with regard to jurisdictional claims in published maps and institutional affiliations. 